\title{
Forfaits liés aux prestations en réadaptation: une utopie?
}

\section{Pierre-François Cuénoud}

Dr, vice-président de la FMH, responsable du département Tarifs et économie de la santé pour les médecins hospitaliers

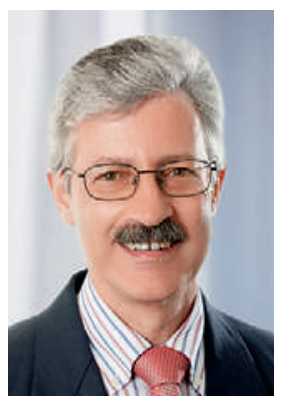

La réforme législative entrée en vigueur en 2009 impose une rétribution des séjours hospitaliers par des forfaits liés aux prestations, quelle que soit la nature du traitement. Grâce à l'expérience des pays qui nous entourent en la matière et suite à une bonne préparation, une structure tarifaire correspondante a pu être élaborée à temps dans le domaine aigu somatique. En revanche, la tâche est nettement plus complexe pour la réadaptation, vu l'absence de repères comparatifs. Pour ne pas interférer avec les décisions thérapeutiques, un système tarifaire doit refléter les enjeux et les charges principaux de la discipline concernée. Contrairement aux pathologies aiguës, la réadaptation intervient de manière moins schématique, prenant en charge un patient dans un état donné pour lui offrir une amélioration la plus significative possible. Le temps nécessaire à ce processus de même que les moyens à déployer pour y parvenir sont extrêmement variables. Pour pouvoir les refléter au mieux, il faut donc s'affranchir des classiques diagnostics et procédures pour appréhender, de manière dynamique, les progrès fonctionnels réalisés.

\section{ST Reha devra permettre une rémunération correcte de tous les types de réadaptation, en particulier concernant la rééducation polyvalente des patients polymorbides.}

Depuis plusieurs années, une nouvelle structure tarifaire pour la réadaptation hospitalière, appelée ST Reha, est en voie d'élaboration sous l'égide de SwissDRG SA. Dans cette phase initiale, des critères d'évaluation fonctionnelle ont été relevés uniquement dans quatre des types de réadaptation recensés, focalisés sur un organe ou un système. Ces travaux, déjà complexes en la forme, ont abouti à l'établissement d'une première version de travail de ST Reha (cf. l'article de P. Ingenpass et B. Rohner à la page 634).

Afin de jouir d'une bonne crédibilité, ST Reha devra permettre une rémunération correcte de tous les types de réadaptation. En particulier, la rééducation polyvalente des patients polymorbides, pour laquelle la Romandie a une sensibilité particulière, devra y trouver sa place. De même, les changements d'orientation en cours de traitement, qui sont la philosophie même de ce type d'hospitalisation, seront à prendre en compte. Au cours des dernières décennies, les modalités d'exécution de la réadaptation ont fortement évolué. Les

\section{Il ne s'agit nullement d'une utopie, mais} le chemin est encore long.

séjours de plusieurs semaines dans une clinique spécialisée, généralement située en altitude, sont peu à peu remplacés par des programmes ambulatoires près du domicile ou du lieu de travail des patients. Il faudra donc veiller à éviter des incitations financières erronées dans les rétributions correspondantes afin de garantir la possibilité d'un choix médical du mode de traitement.

Les forfaits liés aux prestations peuvent être appliqués dans le domaine de la réadaptation. Il ne s'agit nullement d'une utopie, mais le chemin est encore long. La FMH s'engage dans tous les groupes de travail concernés, veillant à ce que le nouveau système n'impose pas de saisie superflue de données, mais plutôt des évaluations également utiles à l'orientation des traitements ainsi qu'à la documentation des dossiers médicaux. Allègement administratif des médecins, primauté du bien-être du patient et adéquation de la rémunération sont les maîtres-mots de notre action. 